\title{
Laminins Regulate Placentation and Pre-eclampsia: Focus on Trophoblasts and Endothelial Cells
}

\author{
Min Liu, Yangxue Yin, Hongbiao Yu and Rong Zhou* \\ Department of Obstetrics and Gynecology, West China Second University Hospital, Sichuan University; Key Laboratory \\ of Birth Defects and Related Diseases of Women and Children, Sichuan University, Ministry of Education, Chengdu, China
}

OPEN ACCESS

Edited by:

Xavier Figueroa,

Pontificia Universidad Católica de Chile, Chile

Reviewed by:

Luis Sobrevia,

Pontificia Universidad Católica

de Chile, Chile

Caroline Dunk

Lunenfeld-Tanenbaum Research

Institute, Canada

*Correspondence:

Rong Zhou

zhourong_hx@scu.edu.cn

Specialty section:

This article was submitted to

Cellular Biochemistry,

a section of the journal

Frontiers in Cell and Developmental

Biology

Received: 11 April 2020

Accepted: 20 July 2020

Published: 07 August 2020

Citation:

Liu M, Yin Y, Yu H and Zhou R

(2020) Laminins Regulate

Placentation and Pre-eclampsia:

Focus on Trophoblasts

and Endothelial Cells.

Front. Cell Dev. Biol. 8:754.

doi: 10.3389/fcell.2020.00754
Pre-eclampsia is a systemic vascular disease characterized by new-onset hypertension and/or proteinuria at $\geq 20$ weeks of gestation and leads to high rates of maternal and perinatal morbidity and mortality. Despite the incomplete understanding of pre-eclampsia pathophysiology, it is accepted that insufficient spiral artery remodeling and endothelial dysfunction are major contributors. Laminins (LNs) are a vital family of extracellular matrix (ECM) molecules present in basement membranes that provide unique spatial and molecular information to regulate implantation and placentation. LNs interact with cell surface receptors to trigger intracellular signals that affect cellular behavior. This mini-review summarizes the role of LNs in placental development during normal pregnancy. Moreover, it describes how LN deficiency can lead to the pre-eclampsia, which is associated with trophoblast and vascular endothelial dysfunction. New research directions and the prospect of clinical diagnosis of $L N$ deficiency are discussed, and the gaps in basic and clinical research in this field are highlighted.

Keywords: pre-eclampsia, laminin, trophoblast, endothelial dysfunction, placenta

\section{INTRODUCTION}

Pre-eclampsia, is a pregnancy disease mainly characterized by gestational hypertension ( $\geq 140 / 90 \mathrm{mmHg}$ ) at gestation $\geq 20$ weeks (Homer et al., 2008); it affects $3-5 \%$ of pregnancies and is more common in low- and middle-income countries (Ananth et al., 2013; Mol et al., 2016). Once diagnosed, the only effective treatment is cesarean delivery. Even if other appropriate treatment is administered for in pregnancy hypertensive diseases, there are still long-term effect on the children and mothers (Mate et al., 2019). Pre-eclampsia is a placental disease with two stages. The first is placental structure abnormality characterized by insufficient spiral artery remodeling and reduced placental perfusion. During a normal pregnancy, placentation begins with the recognition and adhesion of the trophectoderm to the uterine epithelium; trophoblast stem cells originate from the embryonic trophectoderm and differentiate into various trophoblast lineages [as reviewed in Silva and Serakides (2016)]. A recent review (Aplin et al., 2020) clearly summarized the steps in normal and failed uterine spiral arterial conversion. Cytotrophoblast cells invade into the decidua (interstitial trophoblast), and then the wall of maternal uterine spiral artery is destroyed, including the endothelium and smooth muscle cell. Extracellular matrix (ECM) components of the vessel wall are replaced with a fibro-fibrinoid structure, within which residual endovascular trophoblast often remains embedded. The remodeled uterine spiral arteries are characterized by lower resistance and higher flow, and they perfuse the placental villi to support 
placental and fetal development (Brosens et al., 2019). However, conversion failure results in poor blood supply to the intervillous spaces (Brosens et al., 2011). The second stage is manifestation of maternal hypertension and proteinuria with systemic endothelial dysfunction. An imbalance between pro- and anti-angiogenic proteins can lead to increased blood pressure and pre-eclampsia in pregnancy. Some vascular endothelial protective factors [e.g., nitric oxide/nitric oxide synthase, vascular endothelial growth factor (VEGF)] are key factors in maintaining blood pressure, vascular endothelial environmental stability, and angiogenesis (Cudmore et al., 2006). Conversely, vascular endothelial damage factors [e.g., soluble fms-like tyrosine kinase 1 (sFlt-1), soluble endoglin (sEng)] exert anti-angiogenetic effects (De Falco, 2012; Graupner et al., 2019). The transition between the two stages is thought to be due to the release of such factors from the abnormally developed placenta into maternal circulation.

Normal placentation depends on cell-to-cell and cell-to-ECM interactions. The ECM consists of several unmodified and conjugated proteins such as collagen, fibronectin, laminins (LNs) and others that provide a microenvironment for placental cells and regulate cell functions including proliferation, migration, invasion, and signaling (Kim et al., 2014; Graubner et al., 2018). LNs are ECM molecules that comprise a family of glycoproteins found predominantly in basement membranes; they interact with cell surface receptors that transmit intracellular signals that regulate cellular behavior (Lala et al., 2012). Some studies have shown that LN deficiency is associated with many human diseases (McGowan and Marinkovich, 2000). Animal and human studies have shown that LNs are involved in placental development and affect the function of cytotrophoblastic cells (Kuo et al., 2018). This review outlines the critical role of LNs in placental development and molecular mechanisms involved in pre-eclampsia development.

\section{STRUCTURE AND FUNCTION OF LAMININ}

As shown in Figure 1, the basement membrane is the thin sheet of ECM that underlies epithelial and endothelial cells and surrounds muscle cells, Schwann cells, and connective tissue. LNs are ECM molecules that comprise a family of glycoproteins found predominantly in basement membranes, as a trimeric protein containing five $\alpha$, three $\beta$, and three $\gamma$ chains that are genetically distinct (Domogatskaya et al., 2012). The trimers are named according to their composition of $\alpha, \beta$, and $\gamma$ chains. For example, LN-523 is made of $\alpha 5, \beta 2$, and $\gamma 3$ chains (Tzu and Marinkovich, 2008).

Laminins influence cell adhesion, migration, phenotype maintenance, survival, and differentiation (Durbeej, 2010). The LN 33 chain mediates apoptosis, proliferation, invasion, and metastasis of pancreatic cancer cells via the phosphoinositide 3-kinase (PI3K)/Akt signaling pathway (Zhang et al., 2019). Difficulties in isolating LN isoforms from tissues have hampered studies of their biological roles, and most information has come from research on diseases caused by gene mutations or using knockout mice. For instance, LN $\alpha 5$ gene mutation leads to defects in neural tube closure, finger separation, placentation, kidney formation, pulmonary lobe separation, hair morphogenesis, and intestinal smooth muscle differentiation (Miner and Li, 2000; Nguyen et al., 2002; Bolcato-Bellemin et al., 2003). Integrins, dystroglycan, syndecans, and Lutheran/basal cell adhesion molecule (BCAM) are cellular receptors for LNs (Desgrosellier and Cheresh, 2010; Aumailley, 2018; Jin et al., 2019). The biological effects of LNs are presumably largely mediated by surface receptors that link LN matrices to intracellular signaling pathways [as reviewed in Durbeej (2010)]. One group suggested that LNs promote the differentiation and proliferation of rat embryonic stem cells into cardiomyocytes by interacting with the integrin pathway (Wang et al., 2019). Lutheran/BCAM is oncogenic in human urothelial cancers in the presence of its ligands LN-511, LN-521 (Chang et al., 2017).

\section{LAMININS AND PLACENTATION}

Laminin is the basement membrane component that initially promotes cell adhesion and angiogenesis in early embryonic development (Givant-Horwitz et al., 2004). LN-111 affects cell-cell adhesion by changing the localization of vascular endothelial cadherin (Miner et al., 2004). LN activity is closely correlated with the blastocyst implantation window. LN levels in the basement membrane of the placental villi are decreased in late pregnancy compared to early pregnancy, due to the completion of the adhesion and infiltration process of placental trophoblasts (Turpeenniemi-Hujanen et al., 1992). Trophectoderm defects can lead to improper embryo implantation, and abnormal placental formation has deleterious effects on later stages of pregnancy, potentially causing conditions such as pre-eclampsia and intrauterine growth restriction (RedHorse et al., 2004). Thus, LN is essential for proper embryo implantation and subsequent placental formation. One study showed that cytotrophoblast invasion was evident in some LN $\beta 1^{-/-}$mouse embryos, but the extent of invasion was greatly reduced compared with normal embryos (Miner et al., 2004). Moreover, cytotrophoblast behavior and differentiation may be affected by interactions with LN-specific isoforms, thereby regulating the structural formation of the placenta, and attachment of invasive trophoblasts to the basement membrane can trigger the expression of matrix metalloproteinases (MMPs) necessary for their separation from the basement membrane, degradation, and migration (Ramos et al., 1990). LN knockout (LN $\alpha 1, L N \beta 1, L N \beta 5)$ is embryonic lethal in mice. During placental development, LN $\alpha 5$ knockout mice exhibit malformed placental labyrinths and abnormal trophoblast differentiation (Miner et al., 1998; Sekiguchi and Yamada, 2018). In addition, Pijnenborg et al. (1996) described a contribution of LNs to fibrinoid deposition within physiologically remodeled spiral arteries in baboons. Collectively, these findings indicate that LN is particularly important for the subsequent differentiation of cytotrophoblast cells and spiral artery remodeling.

Laminin activates cellular surface receptors to assist in its biological functions, and most recent studies have focused on integrins and LN-111 receptor (LR1). LNs and integrins differ 
A



B
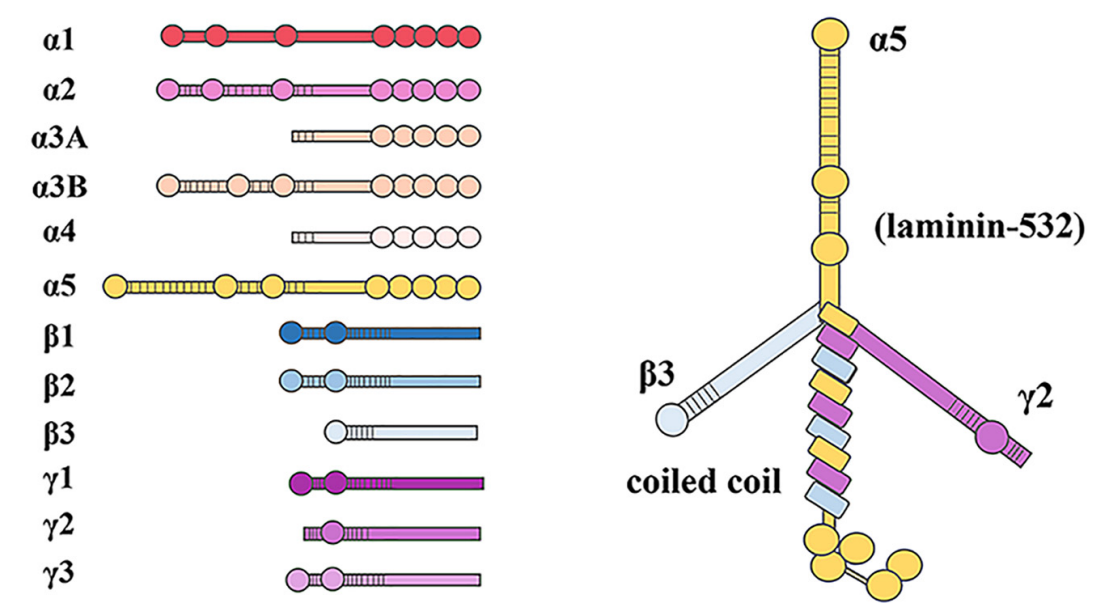

FIGURE 1 | Basement membrane constituents in vessels and LN structure. (A) In blood vessels, the basement membrane separates endothelial cells from supporting cells such as pericytes and connective tissue. The basement membrane consists of LN, type IV collagen, perlecan, and nidogen. (B) LN as a trimeric protein containing five $\alpha$, three $\beta$, and three $\gamma$ chains; all laminin chains share a common domain structure with a number of globular and rod-like domains.

in their binding specificities and affinities (typically including integrins $\alpha 3 \beta 1, \alpha 6 \beta 1, \alpha 7 \beta 1$, and $\alpha 6 \beta 4$ ), and integrin expression patterns are strictly regulated during placental development (Maltepe and Fisher, 2015). For example, integrins $\alpha 6 \beta 1$ and $\alpha 7 \beta 1$ bind to LNs and are abundant in the basement membrane of the endometrial epithelium during the implantation window (Merviel et al., 2001). Integrin serves as a master switch for cytotrophoblast transition from a proliferative $(\alpha 6 \beta 4$ and $\alpha 5 \beta 1)$ to an invasive $(\alpha 1 \beta 1)$ phenotype; this provides a foundation for studying pathological conditions characterized by insufficient trophoblast invasion (Kilburn et al., 2000). Increased LR1 expression in decidual cells of partial and complete moles may enable trophoblasts or other cells (e.g., endothelial cells and lymphocytes) to control invasion by modifying the structure of its primary ligand, LN-111 (Nelson et al., 2008; Kurdoglu et al., 2009).

\section{LAMININS AND PRE-ECLAMPSIA}

Trophoblast invasion of the uterine decidua and maternal vascular system is the key to successful embryo implantation and pregnancy, and LNs play a critical role in this multistep process. Despite an incomplete understanding of pre-eclampsia pathophysiology, it is generally though that the primary mechanisms are insufficient spiral artery remodeling and endothelial dysfunction (Gauster et al., 2009). Thus, we mainly analyze the relationship between LNs and pre-eclampsia from the perspective of improper regulation of LNs on biological behaviors of trophoblasts and vascular endothelial function.

\section{Abnormal Biological Functions of Trophoblasts}

In recent years, abnormal trophoblast function has been proposed to play a major role in pre-eclampsia pathogenesis. Potential cause of trophoblast invasion defect in pre-eclampsia including abnormal levels of cytokines, growth factors, placental factors, and interleukins (Moser et al., 2018). The ratio of type IV collagen to LNs is significantly higher in pre-eclamptic placentas compared to controls, most likely reflecting a lower level of LNs in the villi (Risteli et al., 1984). A study of microarray datasets reported that decreased expression of the $\mathrm{LN} \alpha 2$ chain in the basal plate may be involved in the development of pre-eclampsia 
(Winn et al., 2011). Proteomic analysis also showed that significantly lower LN levels in placental trophoblastic cells from pre-eclampsia pregnant women compared to controls, including $\mathrm{LN} \beta 2$ (12.5-fold), $\mathrm{LN} \alpha 5$ (9.1-fold), and $\mathrm{LN} \gamma 1$ chain (5.6-fold) (Ma et al., 2014). Pre-eclampsia placentas exhibit lower $\mathrm{LN} \alpha 5$ chain levels, and this deficiency inhibits proliferation and invasion but promotes cell apoptosis in HTR-8/SVneo cells, possibly through inhibition of the PI3K/AKT/mammalian target of rapamycin (mTOR) signaling (Zhang et al., 2018). Downregulation of $\mathrm{LN} \alpha 4$ also inhibited the proliferation, migration, and invasion of trophoblasts to suppress the expression of vascular factors in HTR-8/SVneo cells (Ji et al., 2019). Moreover, Shan et al. reported significantly lower expression of $\mathrm{LN} \alpha 4$ in human pre-eclampsia placentas compared to control, and $\mathrm{LN} \alpha 4$ plays a crucial role in trophoblast differentiation and invasion (Shan et al., 2015). Such changes in placental LN levels could weaken trophoblast attachment to the underlying basement membrane and also modify villi permeability and exchange properties.

Signal transduction via LRs in normal and malignant trophoblasts influence their adhesion to the basement membrane (Burrows et al., 1995). LR1 is a non-integrin-type receptor for LN-111 that exerts many effects on cell migration, adhesion, differentiation, invasion, signaling, and tumor metastasis. Decreased immunohistochemical staining for LR1 protein in cytotrophoblasts and syncytiotrophoblasts was observed in pre-eclamptic placentas, and decreased LR1 in cytotrophoblasts might lead to shallow trophoblastic invasion (Kurdoglu et al., 2011). LR1 knockdown remarkably suppressed the migration and invasion potential of JEG3 cells by reducing MMP-2 and MMP-9 activities, suggesting a possible pathogenetic mechanism of pre-eclampsia (Wang et al., 2013, 2015). Zhou et al. (1997) reported that trophoblastic invasion of the uterine spiral artery is limited because there is no decrease in integrin $\alpha 6 \beta 4$ and no increase in integrin $\alpha 1 \beta 1$ in pre-eclampsia pregnancies. Another group observed that integrin $\beta 1$ mRNA was decreased in placental tissue from pre-eclampsia subjects, and then verified its involvement in dysregulating trophoblast cell invasion, survival, and angiogenesis in HTR8/Svneo cells (Li et al., 2013). The slightly decreased integrins $\alpha v$ and $\beta 1$ subunits in the cytotrophoblasts may be a structural basis for poor placental perfusion in pre-eclampsia (Vatansever et al., 2003). Collectively, these findings demonstrate that integrin expression pattern are highly regulated during cytotrophoblast differentiation, and alterations in this process are the pathological basis of endovascular trophoblast invasion into the uterine spiral arteries.

\section{Vascular Endothelial Dysfunction}

Maternal endothelial dysfunction caused by placental factors (pro- and anti-angiogenic proteins) has long been considered one of the pathophysiological factors underlying severe pre-eclampsia (Roberts and Hubel, 2009). However, whether angiomodulatory imbalance is a consequence of an underlying pathology that is common to several different types of placental dysfunction is still unclear, as is whether it reflects a specific abnormality of earlier placental development (Aplin et al., 2020). There is now sufficient evidence to suggest that tissue-specific of LNs and their associated signaling regulates cell behavior and angiogenesis [as reviewed in Simon-Assmann et al. (2011)]. Native LN-111 from the human placenta can promote the formation of interconnected vascular networks among human umbilical vein endothelial cells (HUVECs) (Hackethal et al., 2018). LN $\alpha 4$ chain was strongly expressed in endothelial cells in placental villi and decidua, and its expression was decreased in third-trimester pre-eclamptic placentas (Thyboll et al., 2002). Moreover, $\mathrm{LN} \alpha 4$ small-interfering RNA transfection and hypoxia-reoxygenation intervention reduced HUVEC migration and tube formation abilities by activating mitogen-activated kinase signaling (Shan et al., 2015). Down-regulated $\mathrm{LN} \alpha 5$ is observed in pre-eclampsia placentas and inhibits HUVECs proliferation, migration, and angiogenesis through PI3K/AKT/mTOR pathways (Zhang et al., 2020). However, there are no data to indicate that the endothelial-derived $\mathrm{LN} \alpha 5$ chain is involved in initiating the process as its expression occurs in the later stages of angiogenesis (Langen et al., 2017) when it plays critical roles in vascular stability, maturation, and barrier function (Sorokin et al., 1997; Liu et al., 2018). The above findings indicate that LNs are critical factors for endothelial cells to undergo angiogenesis and differentiate into interconnected tubules. LN deficiency may directly lead to abnormal angiogenesis and pathologic placental development. LNs also regulate angiogenesis through different vascular factors. Glomerular endothelial lesions associated with pre-eclampsia are caused by the blocking VEGF/VEGF receptor (VEGFR) and transforming growth factor- $\beta$ /endoglin signaling pathways by sFlt-1 and sEng, respectively, leading to glomerular endothelial pore loss, cell swelling, and proteinuria (Abrass et al., 2006). A lack of $\mathrm{LN} \alpha 3$ in mice or $\mathrm{LN} \beta 3$ in humans leads to similar defects (Hata et al., 2005). Downregulation of $\alpha v \beta 3$ integrin reduces the priming of endothelial cells and tubulogenesis by inhibiting VEGFR activation (Helal-Neto et al., 2016). LN-111 induces expression of pro-angiogenic molecules, such as VEGF and Cxc chemokine receptor 4, thus leading to increased angiogenesis and tumor growth (Mammadova-Bach et al., 2018). These lines of evidence suggest that LNs can directly and indirectly cause vascular endothelial dysfunction and induce severe pre-eclampsia.

The effect of LR signaling on endothelial cell functions is also important. Interaction between $\alpha 6 \beta 1$ integrin and LN-411 might promote endothelial cell migration in vivo, since their expression patterns overlap with those of newly formed capillaries, where endothelial cells are actively migrating (Sixt et al., 2001). Hypoxia transcriptionally upregulates angiogenic integrins $(\alpha v, \beta 1, \beta 3$, and $\beta 5$ ) in microvascular endothelial cells along with promoting migration and tube formation in human microvascular endothelial cells (Befani and Liakos, 2017). However, the relationship between LR-induced endothelial dysfunction and pre-eclampsia has not been investigated in detail.

\section{FUTURE PERSPECTIVES}

To date, most LN signaling studies in pre-eclampsia have focused on trophoblast function, but a few have investigated other aspects. One found that miR-126-3p promoted matrix-dependent 


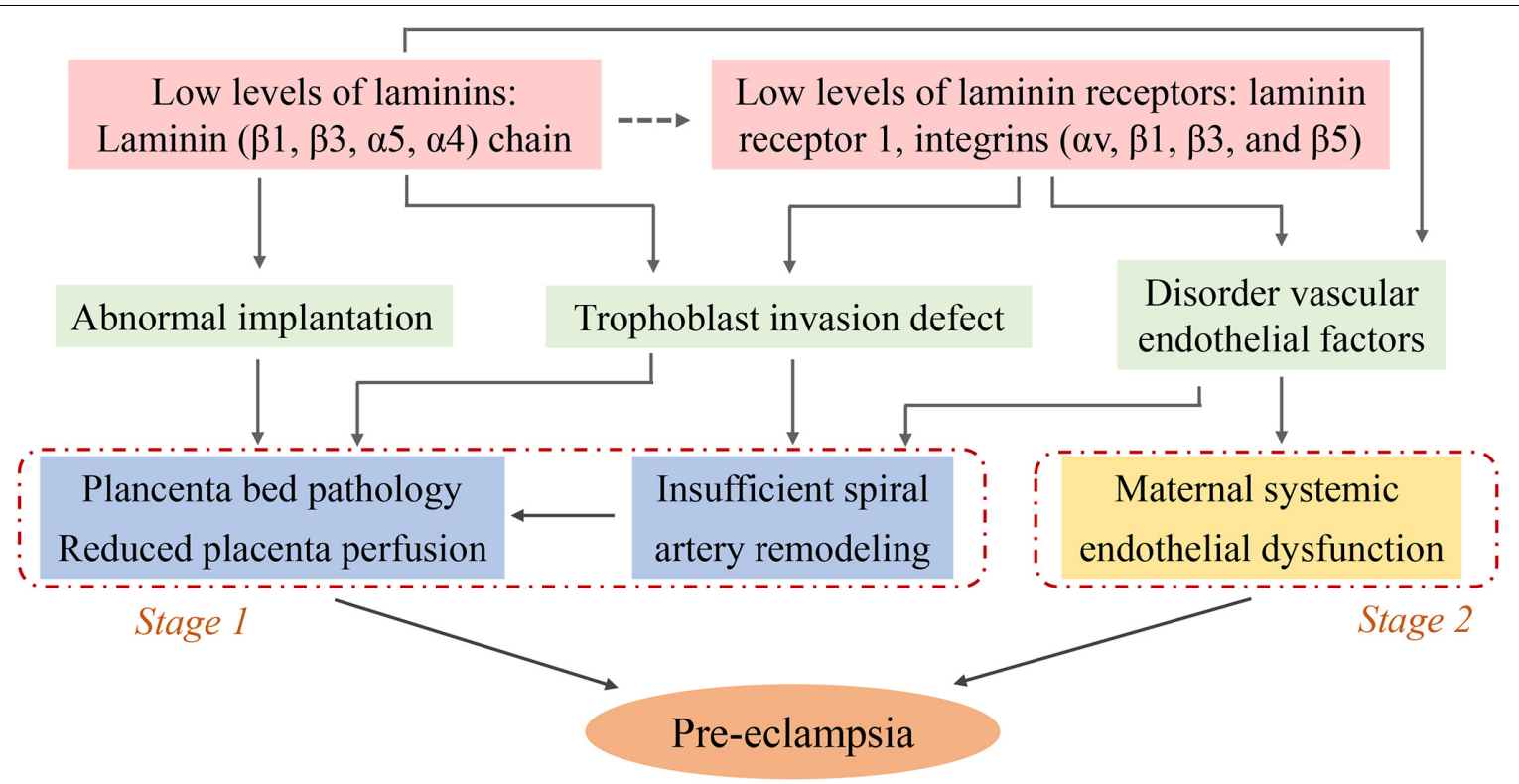

FIGURE 2 | Schematic diagram illustrating the potential causes for the low level of laminins induced- pre-eclampsia: focus on trophoblasts and endothelial cells. The decreased levels of serum LN and placental LN expression (pale pink) contribute to abnormal implantation, trophoblast invasion defect, and disorder vascular endothelial factors (pale green). These pathophysiological states lead to placenta bed pathology, insufficient uterine spiral artery remodeling (blue, known as stage 1 of pre-eclampsia) and maternal systemic endothelial dysfunction (yellow, known as stage 2 of pre-eclampsia). The figure leaves open the possibility that some LNs might play the role by binding to their receptors (dotted arrow, pale pink), while the role of these receptors is clearly known (solid arrow).

perivascular cell attachment, migration, and intercellular interaction (Pitzler et al., 2016). LN-111 is capable of inducing epigenetic changes by inhibiting Dnmtl expression and preventing methylation of the E-cadherin promoter, ultimately promoting metastatic colonization during breast cancer (Benton et al., 2009). However, there is no clear epigenetic mechanism by which LNs regulate occurrence of pre-eclampsia pathogenesis. It is also noteworthy that vascular endothelial cells express only two LN isoforms: LN-411 and LN-511. LN-411 is expressed by all endothelial cells regardless of their developmental stage, and its expression is strongly upregulated by cytokines and growth factors involved in inflammatory events. LN-511 is primarily detectable in endothelial cell basement membranes of capillaries and venules beginning 3-4 weeks postnatal, and its expression is only upregulated by strong proinflammatory signals (Hallmann et al., 2005). Moreover, the ECM can regulate chemokine production in damaged endothelial cells (Zimmerlin et al., 2013). Thus, we hypothesized that LNs expressed by endothelial cells might be involved in the inflammatory response during pre-eclampsia pathogenesis.

Mortality from pre-eclampsia has decreased significantly in the United States due to increased prenatal monitoring and early intervention (Ghossein-Doha et al., 2018). Therefore, it is of considerable significance to explore and identify biomarkers for the detection or control of pre-eclampsia and to develop targeted strategies. Serum or plasma angiogenic factors and cytokines appear to be a reliable risk-stratification approach among women with suspected pre-eclampsia, such as sFlt-1, sEng, placental growth factor (PlGF), interleukin-6, and the sFlt-1/PlGF ratio (Chaiworapongsa et al., 2014). Serum LN level is an important index to assess the degree of liver fibrosis in chronic hepatitis (Lebensztejn et al., 2004). So, could LN be a promising biomarker for diagnosing pre-eclampsia? There is a paucity of prospective studies in this filed with both large sample sizes and well-defined, strict inclusion and exclusion criteria. Furthermore, it is necessary to develop specific and sensitive assays for clinical measurement of LNs.

\section{CONCLUSION}

Laminins play a critical role in pre-eclampsia as demonstrated by a series of clinical and basic studies. Serum LN levels and placental LN expression are decreased in pre-eclamptic pregnancies. Low LN levels are able to induce abnormal biological functions of placental trophoblast cells and vascular endothelial dysfunction, which lead to implantation failure, inappropriate placental spiral artery remodeling and placental vascular injury, these are the main pathological bases for pre-eclampsia. Moreover LRs, especially integrin-type receptor-mediated signaling pathways may be important contributors (summarized in Figure 2). However, the specific molecular mechanisms are complex and unclear, and a more detailed understanding of the signaling mechanisms is needed. This review proposed a possible mechanism of altered LN-induced pre-eclampsia, including epigenetic modifications, cytokines, and inflammatory events. Finally, LNs are promising diagnostic and prognostic biomarkers for this disease. Additional studies can help guide the use of LN clinical diagnosis and identify prevention and treatment targets for pre-eclampsia. 


\section{AUTHOR CONTRIBUTIONS}

ML and RZ designed the outline of the review and wrote the initial manuscript. All authors edited and approved the final manuscript.

\section{REFERENCES}

Abrass, C. K., Berfield, A. K., Ryan, M. C., Carter, W. G., and Hansen, K. M. (2006). Abnormal development of glomerular endothelial and mesangial cells in mice with targeted disruption of the lama3 gene. Kidney Int. 70, 1062-1071. doi: $10.1038 /$ sj.ki.5001706

Ananth, C. V., Keyes, K. M., and Wapner, R. J. (2013). Pre-eclampsia rates in the United States, 1980-2010: age-period-cohort analysis. BMJ 347:f6564. doi: 10.1136/bmj.f6564

Aplin, J. D., Myers, J. E., Timms, K., and Westwood, M. (2020). Tracking placental development in health and disease. Nat. Rev. Endocrinol. 1-16. doi: 10.1038/ s41574-020-0372-6

Aumailley, M. (2018). Isolation and analysis of laminins. Methods Cell Biol. 143, 187-205. doi: 10.1016/bs.mcb.2017.08.011

Befani, C., and Liakos, P. (2017). Hypoxia upregulates integrin gene expression in microvascular endothelial cells and promotes their migration and capillary-like tube formation. Cell Biol. Int. 41, 769-778. doi: 10.1002/cbin.10777

Benton, G., Crooke, E., and George, J. (2009). Laminin-1 induces E-cadherin expression in 3-dimensional cultured breast cancer cells by inhibiting DNA methyltransferase 1 and reversing promoter methylation status. FASEB J. 23, 3884-3895. doi: 10.1096/fj.08-128702

Bolcato-Bellemin, A. L., Lefebvre, O., Arnold, C., Sorokin, L., Miner, J. H., Kedinger, M., et al. (2003). Laminin alpha5 chain is required for intestinal smooth muscle development. Dev. Biol. 260, 376-390. doi: 10.1016/s00121606(03)00254-9

Brosens, I., Pijnenborg, R., Vercruysse, L., and Romero, R. (2011). The "great obstetrical syndromes" are associated with disorders of deep placentation. Am. J. Obstet. Gynecol. 204, 193-201. doi: 10.1016/j.ajog.2010.08.009

Brosens, I., Puttemans, P., and Benagiano, G. (2019). Placental bed research: I. The placental bed: from spiral arteries remodeling to the great obstetrical syndromes. Am. J. Obstet. Gynecol. 221, 437-456. doi: 10.1016/j.ajog.2019. 05.044

Burrows, T. D., King, A., Smith, S. K., and Loke, Y. W. (1995). Human trophoblast adhesion to matrix proteins: inhibition and signal transduction. Hum. Reprod. 10, 2489-2500. doi: 10.1093/oxfordjournals.humrep.a136329

Chaiworapongsa, T., Chaemsaithong, P., Yeo, L., and Romero, R. (2014). Preeclampsia part 1: current understanding of its pathophysiology. Nat. Rev. Nephrol. 10, 466-480. doi: 10.1038/nrneph.2014.102

Chang, H. Y., Chang, H. M., Wu, T. J., Chaing, C. Y., Tzai, T. S., Cheng, H. L., et al. (2017). The role of Lutheran/basal cell adhesion molecule in human bladder carcinogenesis. J. Biomed. Sci. 24:61. doi: 10.1186/s12929-0170360-x

Cudmore, M., Ahmad, S., Al-Ani, B., Hewett, P., Ahmed, S., and Ahmed, A. (2006). VEGF-E activates endothelial nitric oxide synthase to induce angiogenesis via cGMP and PKG-independent pathways. Biochem. Biophys. Res. Commun. 345, 1275-1282. doi: 10.1016/j.bbrc.2006.04.031

De Falco, S. (2012). The discovery of placenta growth factor and its biological activity. Exp. Mol. Med. 44, 1-9. doi: 10.3858/emm.2012.44.1.025

Desgrosellier, J. S., and Cheresh, D. A. (2010). Integrins in cancer: biological implications and therapeutic opportunities. Nat. Rev. Cancer 10, 9-22. doi: $10.1038 / \mathrm{nrc} 2748$

Domogatskaya, A., Rodin, S., and Tryggvason, K. (2012). Functional diversity of laminins. Annu. Rev. Cell Dev. Biol. 28, 523-553. doi: 10.1146/annurev-cellbio101011-155750

Durbeej, M. (2010). Laminins. Cell Tissue Res. 339, 259-268. doi: 10.1007/s00441009-0838-2

Gauster, M., Moser, G., Orendi, K., and Huppertz, B. (2009). Factors involved in regulating trophoblast fusion: potential role in the development of preeclampsia. Placenta 30(Suppl. A), S49-S54. doi: 10.1016/j.placenta.2008. 10.011

\section{FUNDING}

This work was supported by the National Natural Science Foundation of China (Nos. 81571465 and 81871175).

Ghossein-Doha, C., Hooijschuur, M. C. E., and Spaanderman, M. E. A. (2018). Pre-Eclampsia: a twilight zone between health and cardiovascular Disease? J. Am. Coll. Cardiol. 72, 12-16. doi: 10.1016/j.jacc.2018.04.049

Givant-Horwitz, V., Davidson, B., and Reich, R. (2004). Laminin-induced signaling in tumor cells: the role of the M(r) 67,000 laminin receptor. Cancer Res. 64, 3572-3579. doi: 10.1158/0008-5472.CAN-03-3424

Graubner, F. R., Boos, A., Aslan, S., Kucukaslan, I., and Kowalewski, M. P. (2018). Uterine and placental distribution of selected extracellular matrix (ECM) components in the dog. Reproduction 155, 403-421. doi: 10.1530/REP-17-0761

Graupner, O., Karge, A., Flechsenhar, S., Seiler, A., Haller, B., Ortiz, J. U., et al. (2019). Role of sFlt-1/PlGF ratio and feto-maternal Doppler for the prediction of adverse perinatal outcome in late-onset pre-eclampsia. Arch. Gynecol. Obstet. 301, 375-385. doi: 10.1007/s00404-019-05365-9

Hackethal, J., Schuh, C., Hofer, A., Meixner, B., Hennerbichler, S., Redl, H., et al. (2018). Human placenta Laminin-111 as a multifunctional protein for tissue engineering and regenerative medicine. Adv. Exp. Med. Biol. 1077, 3-17. doi: 10.1007/978-981-13-0947-2_1

Hallmann, R., Horn, N., Selg, M., Wendler, O., Pausch, F., and Sorokin, L. M. (2005). Expression and function of laminins in the embryonic and mature vasculature. Physiol. Rev. 85, 979-1000. doi: 10.1152/physrev.00014.2004

Hata, D., Miyazaki, M., Seto, S., Kadota, E., Muso, E., Takasu, K., et al. (2005). Nephrotic syndrome and aberrant expression of laminin isoforms in glomerular basement membranes for an infant with Herlitz junctional epidermolysis bullosa. Pediatrics 116, e601-e607. doi: 10.1542/peds.2005-0160

Helal-Neto, E., Brandao-Costa, R. M., Saldanha-Gama, R., Ribeiro-Pereira, C., Midlej, V., Benchimol, M., et al. (2016). Priming endothelial cells with a melanoma-derived extracellular matrix triggers the activation of alphavbeta3/VEGFR2 axis. J. Cell Physiol. 231, 2464-2473. doi: 10.1002/jcp. 25358

Homer, C. S., Brown, M. A., Mangos, G., and Davis, G. K. (2008). Non-proteinuric pre-eclampsia: a novel risk indicator in women with gestational hypertension. J. Hypertens. 26, 295-302. doi: 10.1097/HJH.0b013e3282fla953

Ji, Y., Zhou, L., Wang, G., Qiao, Y., Tian, Y., and Feng, Y. (2019). Role of LAMA4 gene in regulating extravillous trophoblasts in pathogenesis of preeclampsia. Med. Sci. Monit. 25, 9630-9636. doi: 10.12659/MSM.917402

Jin, S. H., Kim, S. K., and Lee, S. B. (2019). M. leprae interacts with the human epidermal keratinocytes, neonatal (HEKn) via the binding of laminin 5 with alpha-dystroglycan, integrin-beta1, or -beta4. PLoS Negl. Trop. Dis. 13:e0007339. doi: 10.1371/journal.pntd.0007339

Kilburn, B. A., Wang, J., Duniec-Dmuchowski, Z. M., Leach, R. E., Romero, R., and Armant, D. R. (2000). Extracellular matrix composition and hypoxia regulate the expression of HLA-G and integrins in a human trophoblast cell line. Biol. Reprod. 62, 739-747. doi: 10.1095/biolreprod62.3.739

Kim, J. H., Jekarl, D. W., Kim, M., Oh, E. J., Kim, Y., Park, I. Y., et al. (2014). Effects of ECM protein mimetics on adhesion and proliferation of chorion derived mesenchymal stem cells. Int. J. Med. Sci. 11, 298-308. doi: 10.7150/ijms.6672

Kuo, C. Y., Guo, T., Cabrera-Luque, J., Arumugasaamy, N., Bracaglia, L., GarciaVivas, A., et al. (2018). Placental basement membrane proteins are required for effective cytotrophoblast invasion in a three-dimensional bioprinted placenta model. J. Biomed. Mater. Res. A 106, 1476-1487. doi: 10.1002/jbm.a.36350

Kurdoglu, M., Bayram, I., Kolusari, A., Erten, R., Adali, E., Bulut, G., et al. (2009). Expression of laminin receptor 1 in gestational trophoblastic diseases and normal placenta and its relationship with the development of postmolar tumors. Gynecol. Oncol. 114, 306-309. doi: 10.1016/j.ygyno.2009.05.010

Kurdoglu, M., Kurdoglu, Z., Ozen, S., Kucukaydin, Z., Bulut, G., Erten, R., et al. (2011). Expression of laminin receptor 1 in human placentas from normal and preeclamptic pregnancies and its relationship with the severity of preeclampsia. J. Perinat. Med. 39, 411-416. doi: 10.1515/JPM.2011.024

Lala, N., Girish, G. V., Cloutier-Bosworth, A., and Lala, P. K. (2012). Mechanisms in decorin regulation of vascular endothelial growth factor-induced human 
trophoblast migration and acquisition of endothelial phenotype. Biol. Reprod. 87:59. doi: 10.1095/biolreprod.111.097881

Langen, U. H., Pitulescu, M. E., Kim, J. M., Enriquez-Gasca, R., Sivaraj, K. K., Kusumbe, A. P., et al. (2017). Cell-matrix signals specify bone endothelial cells during developmental osteogenesis. Nat. Cell Biol. 19, 189-201. doi: 10.1038/ ncb3476

Lebensztejn, D. M., Kaczmarski, M., Sobaniec-Lotowska, M., Bauer, M., Voelker, M., and Schuppan, D. (2004). Serum laminin-2 and hyaluronan predict severe liver fibrosis in children with chronic hepatitis B. Hepatology 39, 868-869. doi: 10.1002/hep.20147

Li, P., Guo, W., Du, L., Zhao, J., Wang, Y., Liu, L., et al. (2013). microRNA$29 \mathrm{~b}$ contributes to pre-eclampsia through its effects on apoptosis, invasion and angiogenesis of trophoblast cells. Clin. Sci. 124, 27-40. doi: 10.1042/ CS20120121

Liu, G., Wu, R., Yang, B., Deng, C., Lu, X., Walker, S. J., et al. (2018). Human Urinederived stem cell differentiation to endothelial cells with barrier function and nitric oxide production. Stem Cells Transl. Med. 7, 686-698. doi: 10.1002/sctm. 18-0040

Ma, K., Jin, H., Hu, R., Xiong, Y., Zhou, S., Ting, P., et al. (2014). A proteomic analysis of placental trophoblastic cells in preeclampsia-eclampsia. Cell Biochem. Biophys. 69, 247-258. doi: 10.1007/s12013-013-9792-4

Maltepe, E., and Fisher, S. J. (2015). Placenta: the forgotten organ. Annu. Rev. Cell Dev. Biol. 31, 523-552. doi: 10.1146/annurev-cellbio- 100814-125620

Mammadova-Bach, E., Rupp, T., Spenle, C., Jivkov, I., Shankaranarayanan, P., Klein, A., et al. (2018). Laminin alphal orchestrates VEGFA functions in the ecosystem of colorectal carcinoma. Biol. Cell. 110, 178-195. doi: 10.1111/boc. 201800007

Mate, A., Blanca, A. J., Salsoso, R., Toledo, F., Stiefel, P., Sobrevia, L., et al. (2019). Insulin therapy in pregnancy hypertensive diseases and its effect on the offspring and mother later in life. Curr. Vasc. Pharmacol. 17, 455-464. doi: 10.2174/1570161117666181114125109

McGowan, K. A., and Marinkovich, M. P. (2000). Laminins and human disease. Microsc. Res. Tech. 51, 262-279. doi: 10.1002/1097-0029(20001101)51:3<262:: aid-jemt6>3.0.co;2-v

Merviel, P., Challier, J. C., Carbillon, L., Foidart, J. M., and Uzan, S. (2001). The role of integrins in human embryo implantation. Fetal Diagn. Ther. 16, 364-371. doi: $10.1159 / 000053942$

Miner, J. H., Cunningham, J., and Sanes, J. R. (1998). Roles for laminin in embryogenesis: exencephaly, syndactyly, and placentopathy in mice lacking the laminin alpha5 chain. J. Cell Biol. 143, 1713-1723. doi: 10.1083/jcb.143.6.1713

Miner, J. H., and Li, C. (2000). Defective glomerulogenesis in the absence of laminin alpha5 demonstrates a developmental role for the kidney glomerular basement membrane. Dev. Biol. 217, 278-289. doi: 10.1006/dbio.1999.9546

Miner, J. H., Li, C., Mudd, J. L., Go, G., and Sutherland, A. E. (2004). Compositional and structural requirements for laminin and basement membranes during mouse embryo implantation and gastrulation. Development 131, 2247-2256. doi: $10.1242 /$ dev.01112

Mol, B. W. J., Roberts, C. T., Thangaratinam, S., Magee, L. A., de Groot, C. J. M., and Hofmeyr, G. J. (2016). Pre-eclampsia. Lancet 387, 999-1011. doi: 10.1016/ S0140-6736(15)00070-77

Moser, G., Windsperger, K., Pollheimer, J., de Sousa Lopes, S. C., and Huppertz, B. (2018). Human trophoblast invasion: new and unexpected routes and functions. Histochem. Cell Biol. 150, 361-370. doi: 10.1007/s00418-018-1699-0

Nelson, J., McFerran, N. V., Pivato, G., Chambers, E., Doherty, C., Steele, D., et al. (2008). The $67 \mathrm{kDa}$ laminin receptor: structure, function and role in disease. Biosci. Rep. 28, 33-48. doi: 10.1042/BSR20070004

Nguyen, N. M., Miner, J. H., Pierce, R. A., and Senior, R. M. (2002). Laminin alpha 5 is required for lobar septation and visceral pleural basement membrane formation in the developing mouse lung. Dev. Biol. 246, 231-244. doi: 10.1006/ dbio.2002.0658

Pijnenborg, R., D’Hooghe, T., Vercruysse, L., and Bambra, C. (1996). Evaluation of trophoblast invasion in placental bed biopsies of the baboon, with immunohistochemical localisation of cytokeratin, fibronectin, and laminin. J. Med. Primatol. 25, 272-281. doi: 10.1111/j.1600-0684.1996.tb00210.x

Pitzler, L., Auler, M., Probst, K., Frie, C., Bergmeier, V., Holzer, T., et al. (2016). miR-126-3p promotes matrix-dependent perivascular cell attachment, migration and intercellular interaction. Stem Cells 34, 1297-1309. doi: 10.1002/ stem. 2308
Ramos, D. M., Berston, E. D., and Kramer, R. H. (1990). Analysis of integrin receptors for laminin and type IV collagen on metastatic B16 melanoma cells. Cancer Res. 50, 728-734.

Red-Horse, K., Zhou, Y., Genbacev, O., Prakobphol, A., Foulk, R., McMaster, M., et al. (2004). Trophoblast differentiation during embryo implantation and formation of the maternal-fetal interface. J. Clin. Invest. 114, 744-754. doi: 10.1172/JCI22991

Risteli, J., Foidart, J. M., Risteli, L., Boniver, J., and Goffinet, G. (1984). The basement membrane proteins laminin and type IV collagen in isolated villi in pre-eclampsia. Placenta 5, 541-550. doi: 10.1016/s0143-4004(84)80008-9

Roberts, J. M., and Hubel, C. A. (2009). The two stage model of preeclampsia: variations on the theme. Placenta 30(Suppl. A), S32-S37. doi: 10.1016/j. placenta.2008.11.009

Sekiguchi, R., and Yamada, K. M. (2018). Basement membranes in development and disease. Curr. Top. Dev. Biol. 130, 143-191. doi: 10.1016/bs.ctdb.2018. 02.005

Shan, N., Zhang, X., Xiao, X., Zhang, H., Chen, Y., Luo, X., et al. (2015). The role of laminin alpha4 in human umbilical vein endothelial cells and pathological mechanism of preeclampsia. Reprod. Sci. 22, 969-979. doi: 10. $1177 / 1933719115570913$

Silva, J. F., and Serakides, R. (2016). Intrauterine trophoblast migration: a comparative view of humans and rodents. Cell Adh. Migr. 10, 88-110. doi: 10.1080/19336918.2015.1120397

Simon-Assmann, P., Orend, G., Mammadova-Bach, E., Spenle, C., and Lefebvre, O. (2011). Role of laminins in physiological and pathological angiogenesis. Int. J. Dev. Biol. 55, 455-465. doi: 10.1387/ijdb.103223ps

Sixt, M., Engelhardt, B., Pausch, F., Hallmann, R., Wendler, O., and Sorokin, L. M. (2001). Endothelial cell laminin isoforms, laminins 8 and 10, play decisive roles in $\mathrm{T}$ cell recruitment across the blood-brain barrier in experimental autoimmune encephalomyelitis. J. Cell Biol. 153, 933-946. doi: 10.1083/jcb.153. 5.933

Sorokin, L. M., Pausch, F., Frieser, M., Kroger, S., Ohage, E., and Deutzmann, R. (1997). Developmental regulation of the laminin alpha5 chain suggests a role in epithelial and endothelial cell maturation. Dev. Biol. 189, 285-300. doi: 10.1006/dbio.1997.8668

Thyboll, J., Kortesmaa, J., Cao, R., Soininen, R., Wang, L., Iivanainen, A., et al. (2002). Deletion of the laminin alpha4 chain leads to impaired microvessel maturation. Mol. Cell Biol. 22, 1194-1202. doi: 10.1128/mcb.22.4.1194-1202. 2002

Turpeenniemi-Hujanen, T., Ronnberg, L., Kauppila, A., and Puistola, U. (1992). Laminin in the human embryo implantation: analogy to the invasion by malignant cells. Fertil. Steril. 58, 105-113. doi: 10.1016/s0015-0282(16)55145-0

Tzu, J., and Marinkovich, M. P. (2008). Bridging structure with function: structural, regulatory, and developmental role of laminins. Int. J. Biochem. Cell Biol. 40, 199-214. doi: 10.1016/j.biocel.2007.07.015

Vatansever, H. S., Inan, V. S., Lacin, S., and Koyuncu, F. (2003). Immunolocalization of alphaV, alpha 3 and betal integrins in the human placenta with pre-eclampsia. Acta Histochem. 105, 253-260. doi: 10.1078/ 0065-1281-00708

Wang, D., Wang, Y., Liu, H., Tong, C., Ying, Q., Sachinidis, A., et al. (2019). Laminin promotes differentiation of rat embryonic stem cells into cardiomyocytes by activating the integrin/FAK/PI3K p85 pathway. J. Cell Mol. Med. 23, 3629-3640. doi: 10.1111/jcmm.14264

Wang, L., Yu, Y., Guan, H., Liu, T., and Qiao, C. (2015). 67-kDa Laminin receptor contributes to hypoxia-induced migration and invasion of trophoblast-like cells by mediating matrix metalloproteinase-9. Clin. Exp. Pharmacol. Physiol. 42, 549-558. doi: 10.1111/1440-1681.12389

Wang, L., Zhang, D., Yu, Y., Guan, H., Qiao, C., and Shang, T. (2013). RNA interference-mediated silencing of laminin receptor 1 (LR1) suppresses migration and invasion and down-regulates matrix metalloproteinase (MMP)2 and MMP-9 in trophoblast cells: implication in the pathogenesis of preeclampsia. J. Mol. Histol. 44, 661-668. doi: 10.1007/s10735-0139515-6

Winn, V. D., Gormley, M., and Fisher, S. J. (2011). The impact of preeclampsia on gene expression at the maternal-fetal interface. Pregnancy Hypertens. 1 100-108. doi: 10.1016/j.preghy.2010.12.001

Zhang, H., Pan, Y. Z., Cheung, M., Cao, M., Yu, C., Chen, L., et al. (2019). LAMB3 mediates apoptotic, proliferative, invasive, and metastatic behaviors in 
pancreatic cancer by regulating the PI3K/Akt signaling pathway. Cell Death Dis. 10:230. doi: 10.1038/s41419-019-1320-z

Zhang, X., Li, Q., Jiang, W., Xiong, X., Li, H., Zhao, J., et al. (2020). LAMA5 promotes human umbilical vein endothelial cells migration, proliferation, and angiogenesis and is decreased in preeclampsia. J. Matern. Fetal Neonatal Med. 33, 1114-1124. doi: 10.1080/14767058.2018.1514597

Zhang, X. M., Xiong, X., Tong, C., Li, Q., Huang, S., Li, Q. S., et al. (2018). Downregulation of laminin (LN)- alpha5 is associated with preeclampsia and impairs trophoblast cell viability and invasiveness through PI3K signaling pathway. Cell Physiol. Biochem. 51, 2030-2040. doi: 10.1159/000495822

Zhou, Y., Damsky, C. H., and Fisher, S. J. (1997). Preeclampsia is associated with failure of human cytotrophoblasts to mimic a vascular adhesion phenotype. One cause of defective endovascular invasion in this syndrome? J. Clin. Invest. 99, 2152-2164. doi: 10.1172/JCI119388
Zimmerlin, L., Park, T. S., Zambidis, E. T., Donnenberg, V. S., and Donnenberg, A. D. (2013). Mesenchymal stem cell secretome and regenerative therapy after cancer. Biochimie 95, 2235-2245. doi: 10.1016/j.biochi.2013.05.010

Conflict of Interest: The authors declare that the research was conducted in the absence of any commercial or financial relationships that could be construed as a potential conflict of interest.

Copyright $\odot 2020 \mathrm{Liu}, \mathrm{Yin}, \mathrm{Yu}$ and Zhou. This is an open-access article distributed under the terms of the Creative Commons Attribution License (CC BY). The use, distribution or reproduction in other forums is permitted, provided the original author(s) and the copyright owner(s) are credited and that the original publication in this journal is cited, in accordance with accepted academic practice. No use, distribution or reproduction is permitted which does not comply with these terms. 